\title{
Morfometría y fecundidad de Profilicollis bullocki Mateo, Córdova \& Guzmán 1982 (Acanthocephala: Polymorphidae) en especies simpátricas de aves costeras de Chile
}

\author{
Morphometry and fecundity of Profilicollis bullocki Mateo, Córdova \& Guzmán 1982 \\ (Acanthocephala: Polymorphidae) in sympatric coastal bird species of Chile
}

\author{
CLAUDIA RIQUELME ${ }^{1}$, MARIO GEORGE-NASCIMENTO*,1 \& LUIS BALBOA ${ }^{2}$ \\ ${ }^{1}$ Departamento de Ecología Costera, Facultad de Ciencias, \\ Universidad Católica de la Santísima Concepción, Casilla 297, Concepción, Chile \\ ${ }^{2}$ Universidad Santo Tomás, Buena Vecindad 91, Puerto Montt, Chile \\ * e-mail para correspondencia: mgeorgen@ucsc.cl
}

\begin{abstract}
RESUMEN
Se describe y se compara la morfología y fecundidad de individuos adultos del acantocéfalo Profilicollis bullocki Mateo, Córdova \& Guzmán 1982 (Acanthocephala: Polymorphidae) extraídos del intestino de cuatro especies de aves costeras Larus dominicanus Lichtenstein 1823, Larus pipixcan Wagler 1831, Podiceps occipitalis Garnot 1826 y Numenius phaeopus Linné 1758, capturadas en Caleta Lenga (36 $45^{\prime}$ S, $\left.73^{\circ} 10^{\prime} \mathrm{O}\right)$, Chile. Los resultados señalan que la identidad de la especie hospedadora es un factor relevante para entender las variaciones de la morfología y de la fecundidad del parásito. Los acantocéfalos recolectados desde $L$. dominicanus y L. pipixcan eran los de mayor tamaño corporal. Además, la fecundidad de los parásitos aumentaba con su tamaño corporal. Sin embargo, el análisis de los residuos de la regresión entre la fecundidad y la longitud total del cuerpo de $P$. bullocki mostró que la fecundidad del parásito en $L$. dominicanus es similar a la encontrada en L. pipixcan y que en estas especies es significativamente mayor que la encontrada en los parásitos recolectados de $P$. occipitalis. Se discute que para establecer qué hospedadores son de mejor calidad para este parásito, aparte de su desempeño reproductivo del parásito en cada especie hospedadora, es necesario también considerar la abundancia de los hospedadores y la magnitud que alcanzan las poblaciones del parásito en cada una de ellas.
\end{abstract}

Palabras clave: morfometría, fecundidad, acantocéfalos, hospedadores simpátricos.

\begin{abstract}
We describe and compare the variations in morphology and fecundity of Profilicollis bullocki Mateo, Córdova \& Guzmán 1982 (Acanthocephala: Polymorphidae) adults collected from 4 alternative sympatric and definitive marine coastal bird host species (Larus dominicanus Lichtenstein 1823, Larus pipixcan Wagler 1831, Podiceps occipitalis Garnot 1826 and Numenius phaeopus Linné 1758), sampled at Caleta Lenga, Chile $\left(36^{\circ} 45^{\prime} \mathrm{S}, 73^{\circ} 10^{\prime} \mathrm{W}\right)$. Results show that the specific identity of the host species is a relevant factor to explain morphometric and reproductive variations of $P$. bullocki. Parasites in Larus spp. attain larger body size than in the two other host species. In addition, fecundity increases with parasite body size. However, analysis on the residuals of the linear regression between fecundity and body size show that fecundity in L. dominicanus and in L. pipixcan are similar and both higher than in P. occipitalis. We suggest that estimates of host abundance along with abundance of parasites within each host species are needed to determine which is the best host for a parasite, as well as the reproductive performance of the parasite in each host species.
\end{abstract}

Key words: morphometry, fecundity, acanthocephalans, sympatric hosts.

\section{INTRODUCCIÓN}

Los atributos morfológicos y los rasgos de historia de vida de los individuos de toda especie son afectados por las condiciones ambientales (Paterson \& Banks 2001). En los organismos de vida parásita, la identidad de la especie de hospedador determina gran parte de las condiciones ambientales en las que ellos viven (Hochberg et al. 1992, Koella et al. 1998). Por esto, los parásitos que poseen un amplio rango de hospedadores se encuentran 
expuestos a diferentes presiones selectivas determinadas por la calidad del ambiente que entrega la especie hospedadora. Por ende, pueden afectar directamente los componentes de la adecuación biológica de los parásitos (fecundidad y sobrevivencia, ver GeorgeNascimento \& Marín 1992). Así, las variaciones en los componentes de la adecuación biológica de los parásitos que son inducidas por la especie hospedadora pueden evidenciarse, indirectamente, a través de su efecto sobre caracteres fenotípicos de los parásitos correlacionados con dichos componentes (Poulin 1998). Distintas especies hospedadoras inducen variaciones morfológicas en el tamaño corporal, proporciones de las distintas partes del cuerpo, forma y tamaño de apéndices, y caracteres sexuales (GeorgeNascimento \& Muñoz 1997, GeorgeNascimento et al. 2004). Sin embargo, no es frecuente que se efectúen comparaciones entre parásitos que provienen de hospedadores definitivos alternativos de un amplio espectro de linajes habitando en condiciones de simpatría (Criscione et al. 2005).

Aunque hay varios estudios en el efecto de la identidad específica de los hospedadores sobre las variaciones de los caracteres morfológicos y reproductivos de los parásitos (Gil De Pertierra et al. 1996, GeorgeNascimento \& Muñoz 1997, GeorgeNascimento et al. 1992, 2004), pocos han utilizado hospedadores de una misma localidad, lo que puede afectar los patrones detectados. De hecho, uno de los aspectos más determinantes de las características morfológicas de los parásitos está dado por la fuente de propágulos (Poulin 1998), lo que puede variar entre localidades (GeorgeNascimento et al. 1992, 2004, GeorgeNascimento \& Llanos 1995, Dudinak \& Snabel 2001). Por otro lado, los caracteres morfológicos de los organismos son usualmente estudiados para el diagnóstico de la identidad específica de un taxon (Crompton 1970, Amin 1985). Sin embargo, varias de las características de los organismos pueden variar debido a las condiciones ambientales. Más aún, en organismos parásitos dichas variaciones pueden aumentar con la distancia taxonómica de los hospedadores (Poulin 1998).

La especie objeto de este estudio es el acantocéfalo Profilicollis bullocki Mateo et al.
1982 (Polymorphidae). El ciclo de vida de las especies de este género se caracteriza por el uso de crustáceos decápodos como hospedadores intermediarios (Nickol et al. 1999, Brockerhoff \& Smales 2002) y de aves marinas como hospedadores definitivos (Crompton \& Nickol 1985). Los crustáceos albergan el estado larval (Nickol et al. 1999) y los hospedadores definitivos son aves marinas (Zdzitowiecki 1985), aunque también son capaces de madurar en mamíferos, incluido el hombre (Hennessy \& Morejohn 1977, Tantaleán et al. 2002).

Esta especie de acantocéfalo ha sido registrada en Larus belcheri (Charadriiformes: Laridae) como Polymorphus (Profilicollis) altmani (ver Mateo et al. 1982, 1983), y como $P$. altmani en L. pipixcan y en L. modestus (ver Tantaleán et al. 2005) en la costa del Perú. Sin embargo, y a pesar de que se reconoce una mayor rigurosidad en la determinación taxonómica realizada por Mateo et al. (1982, 1983), el debate taxonómico persiste (Tantaleán et al. 2002, Tantaleán \& Cárdenas 2004, Tantaleán et al. 2005). Esta especie ha sido encontrada en Chile al estado larval en Emerita analoga (Stimpson 1857) (ver Oliva et al. 1992), y al estado adulto en diversas especies de aves, tales como L. dominicanus, $L$. pipixcan (Charadriiformes: Laridae), Numenius phaeopus (Charadriiformes: Scolopacidae), Podiceps occipitalis (Podicipediformes: Podicipedidae), Egretta sp. (Ciconiformes: Ardeidae; L. Balboa, datos no publicados). Además, hay registros al estado adulto de la especie congenérica $P$. antarcticus Zdzitowiecki 1985 en L. dominicanus al estado adulto, y en la jaiba estuarina Hemigrapsus crenulatus al estado larval (Torres et al. 1991, Pulgar et al. 1995).

La distribución geográfica de $L$. dominicanus abarca todo el hemisferio Sur, circunvala toda la región del polo austral y es abundante en las costas de Chile (Bahamondes \& Castilla 1986). En cambio, L. pipixcan es una especie migratoria que nidifica en la tundra y humedales del Sur de Canadá, y recorre anualmente más de $8.000 \mathrm{~km}$ hasta las costas de Perú y Chile. Por su parte, P. occipitalis es una ave zambullidora que habita la franja de la costa y totoral, y se distribuye a lo largo de la cordillera de los Andes en Sudamérica y en terrenos bajo climas templados de Chile. Finalmente, $N$. phaeopus es una especie 
migratoria que se reproduce en regiones circumpolares del norte de Europa, alcanzando a Tierra del Fuego, sur de Asia y noreste de la India en sus migraciones (Araya \& Miller 1992, Araya et al. 1995).

En este estudio se compara por primera vez el desempeño de $P$. bullocki entre varias especies de hospedadores definitivos alternativos que coexisten en una localidad de la costa de Chile. Se eligió estudiar hospedadores que cohabitaran espacial y temporalmente para obviar las variaciones geográficas, y descartar así su potencial efecto en la interacción parásito-hospedador. Además se compara el efecto de las especies simpátricas de hospedadores definitivos sobre caracteres fenotípicos y en particular en algunos asociados a la adecuación biológica de los parásitos. Finalmente, se estudia si el característico dimorfismo sexual de los acantocéfalos se manifiesta de similar manera entre estas especies de hospedadores definitivos.

\section{MATERIALES Y MÉTODOS}

Entre julio de 2001 y septiembre de 2002, se obtuvieron 122 especímenes de $P$. bullocki desde el intestino de 19 ejemplares de aves acuáticas adultas pertenecientes a cuatro especies (cinco Larus dominicanus, cinco $L$. pipixcan, cinco Podiceps occipitalis y cuatro individuos de Numenius phaeopus), desde la localidad de Caleta Lenga, Chile $\left(36^{\circ} 45^{\prime} \mathrm{S}\right.$, $73^{\circ} 10^{\prime}$ O, Tabla 1). Todos los ejemplares fueron cazados mediante escopeta luego de acceder a los permisos otorgados por la autoridad pertinente. Luego fueron trasladados al laboratorio y congelados a $-20{ }^{\circ} \mathrm{C}$, hasta el momento de su examen. Para extraer los parásitos se procedió a descongelar y disectar cada hospedador a través de un corte ventral para extraer el tracto digestivo. Se revisó el lumen del esófago, molleja, yeyuno, íleon, ciegos, recto y cloaca en la búsqueda de acantocéfalos. Los parásitos encontrados fueron separados del tejido del hospedador cuidando de mantener intactas las estructuras de fijación del parásito y traspasados a agua destilada. Los parásitos utilizados para morfometría fueron fijados con una solución de formalina al $10 \%$ y guardados en frascos individuales. Posteriormente, cada ejemplar fue traspasado a lactofenol de Amman para transparentar sus estructuras internas (Brockerhoff \& Smales 2002).

La determinación taxonómica de $P$. bullocki sigue a Mateo et al. (1982, 1983). Es decir, los caracteres de interés diagnóstico utilizados fueron: el número de hileras longitudinales de la probóscide y el número de ganchos de dos hileras contiguas. Según dichos autores, la probóscide de $P$. bullocki posee 27 a 33 hileras longitudinales con 14 a 15 ganchos por hilera. En cambio, la probóscide de $P$. antarcticus presenta 16 a 22 hileras longitudinales con 8 a 9 ganchos por hilera. Algunos ejemplares determinados como $P$. bullocki fueron seleccionados y deshidratados en una serie de alcoholes, llevados al punto de secado crítico en $\mathrm{CO}_{2}$, y finalmente examinados bajo microscopio electrónico de barrido.

A todos los parásitos se les midieron al menos nueve variables: la longitud del cuerpo, el largo y ancho de la probóscide, el largo y ancho del cuello, el largo y ancho del tronco, el largo de los lemniscos y el largo del receptáculo de la probóscide. En un número mínimo de 8 acantocéfalos hembra por especie de hospedador se midió además el largo y ancho de los huevos (total de 11 variables en las hembras), mientras que en al menos cinco machos provenientes de cada especie de hospedador se midió además el largo y ancho del testículo anterior y posterior y el ancho de las 4 glándulas cemento (total 14 variables en machos). Todas estas medidas se expresan en $\mathrm{mm}$, a no ser que se indique otra unidad.

En el análisis de las variaciones de la morfometría de $P$. bullocki entre las especies hospedadoras, se describieron primero las nueve variables compartidas por todos los ejemplares, mediante análisis de componentes principales (ACP) efectuados sobre la matriz de correlación, y mediante otros dos ACP, uno sobre las 11 variables registradas en las hembras, y otro ACP sobre las 14 registradas en los machos.

El posible dimorfismo sexual de $P$. bullocki fue evaluado con las nueve variables comunes a ambos sexos. Para ello se realizaron pruebas de t de Student sobre los puntajes de las observaciones en los dos primeros ejes derivados del ACP para cada especie hospedadora por separado, con el sexo como variable categórica. 


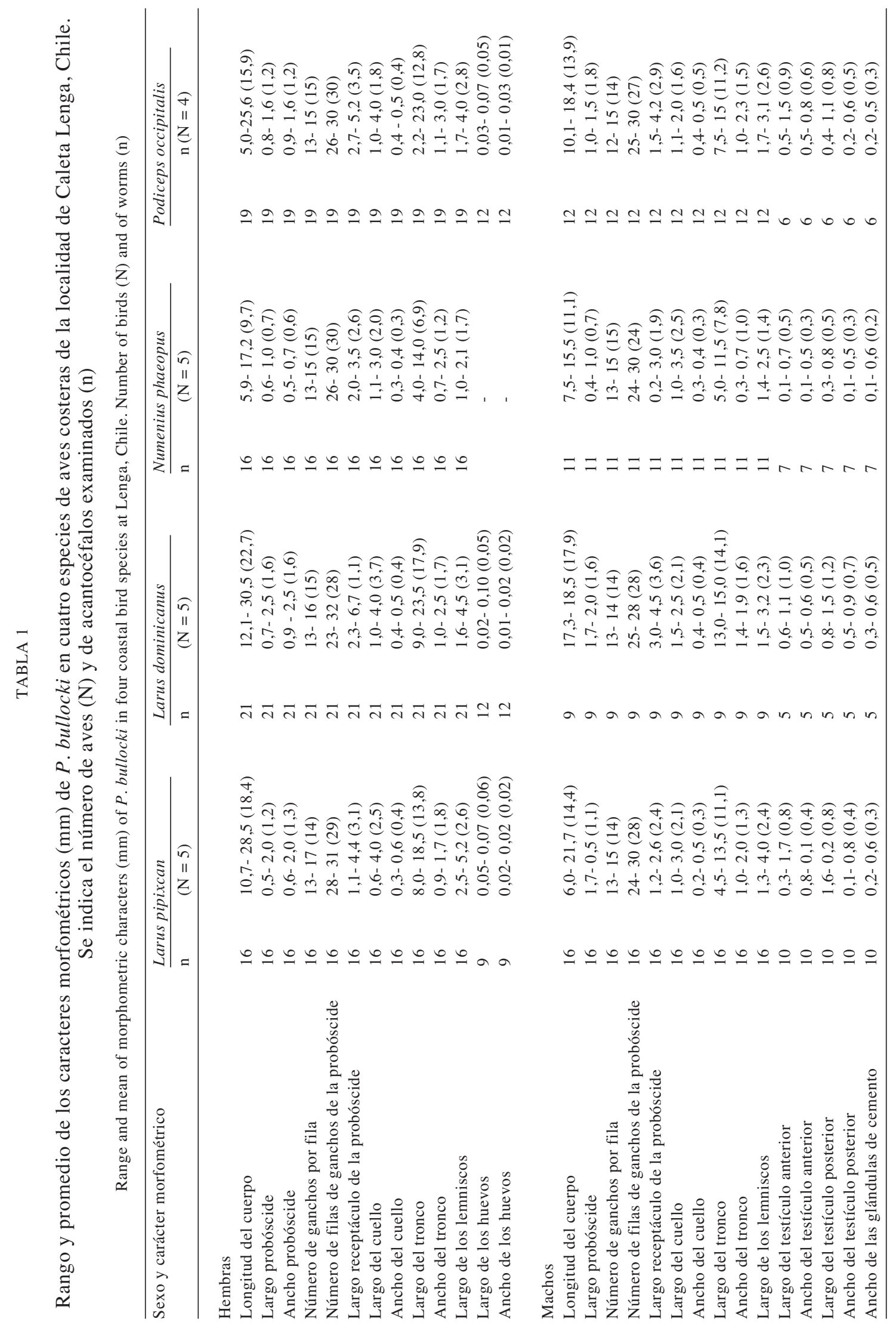


El efecto de la especie hospedadora sobre la morfometría de los acantocéfalos fue evaluado tanto para las variables comunes a ambos sexos, como para cada sexo por separado. Para esto, se efectuaron análisis de la varianza de una vía sobre los puntajes de las observaciones en los dos primeros ejes derivados de cada $\mathrm{ACP}$, con la especie hospedadora como variable clasificatoria. Se usó la prueba a posteriori de Tukey para evaluar la significancia estadística de las distintas especies hospedadoras.

El sexo de cada parásito fue determinado mediante inspección visual bajo microscopio. Las variables morfométricas internas y externas fueron medidas con una reglilla graduada acoplada al ocular de un microscopio y de una lupa respectivamente. Se estimó la fecundidad de 30 hembras maduras (Zdzitowiecki 1991) de $P$. bullocki obtenidas de medir 10 ejemplares provenientes de cada especie de hospedador, excepto de $N$. phaeopus, ya que en esta última especie no se encontraron acantocéfalos hembra con huevos. Para ello, en cada parásito se extrajeron los huevos, los que luego se llevaron a una solución acuosa de $2 \mathrm{~mL}$ para posteriormente homogenizarlos con un agitador magnético. De cada solución se muestrearon, con reemplazo, 10 alícuotas de $0,01 \mathrm{~mL}$ con una micropipeta las que fueron depositadas sobre un portaobjetos que fue examinado bajo un microscopio óptico para el conteo de los huevos. El promedio de las 10 alícuotas fue multiplicado por el factor de conversión al volumen de la muestra (x 200), y se consideró como estimador puntual de la fecundidad de cada hembra (McClelland 1980).

Para evaluar el efecto de la especie hospedadora sobre las variaciones de la fecundidad de las hembras, se realizó una regresión lineal simple entre la fecundidad y el volumen del tronco las hembras de P. bullocki. Posteriormente, sobre los residuos de dicha regresión, se realizó un análisis de la varianza de una vía, y se compararon las medias con la prueba a posteriori de Tukey (Sokal \& Rohlf 1995).

\section{RESULTADOS}

La longitud total del cuerpo de los parásitos fluctuaba entre 9,7 y $22,7 \mathrm{~mm}$ en las cuatro especies hospedadoras, y alcanzaba valores máximos en ambas especies de Larus (Tabla 1).

La morfometría de $P$. bullocki cambiaba significativamente entre especies de hospedadores. El ACP realizado sobre las nueve variables morfométricas comunes a ambos sexos mostró que cerca del $70 \%$ de la varianza estaba explicada por los dos primeros componentes principales (Fig. 1, Tabla 2). Las variables mejor correlacionadas con los puntajes del primer componente principal (58\% varianza) fueron el largo del tronco, la longitud del cuerpo, el ancho del tronco, largo y ancho de la probóscide, mientras que el largo del cuello estaba más asociado a las variaciones del segundo componente principal (12\% de la varianza).

Las tendencias centrales de los puntajes de las observaciones en el primer y segundo componente principal efectuado con las nueve variables comunes a ambos sexos mostraron diferencias significativas entre especies de hospedadores (primer componente: $\mathrm{F}_{3,118}=29,26 ; \mathrm{P}<0,0001$; segundo componente: $\left.\mathrm{F}_{3,118}=3,76 ; \mathrm{P}<0,01\right)$. Las pruebas a posteriori muestran que las variables asociadas al primer componente principal siempre alcanzan valores mayores en $L$. dominicanus y menores en $N$. phaeopus, en tanto que en L. pipixcan y P. occipitalis son de similar magnitud. Estas diferencias no se observaron en el segundo componente principal ya que se encontraron valores similares entre los parásitos de L. dominicanus, L. pipixcan y $N$. phaeopus, aunque significativamente mayores que en $P$. occipitalis $(\mathrm{P}<0,0001)$.

En los ACP hechos sobre cada sexo por separado (Fig. 1) se encontraron resultados similares a los hallados para ambos sexos en conjunto. Primero, entre el 65 y el $66 \%$ de la variación en la morfometría estaba explicada por los dos primeros componentes principales (Tabla 2). Segundo, en ambos sexos ocurría que la longitud total y ancho del tronco, el largo y ancho de la probóscide, y el largo y ancho del testículo posterior, eran las variables que más se correlacionaban con el primer componente principal. En machos, el largo del cuello y el ancho de las glándulas de cemento se asociaban al segundo componente. En hembras, el largo y ancho del cuello se asociaban al segundo componente (Tabla 2). Tercero, los puntajes de las observaciones en el primer componente 
principal resultaron ser significativamente diferentes entre especies hospedadoras, tanto en machos $\left(\mathrm{F}_{3,24}=5,70 ; \mathrm{P}<0,005\right)$, como en hembras $\left(\mathrm{F}_{2,30}=15,07 ; \mathrm{P}<0,0001\right)$. Las pruebas a posteriori muestran valores similares entre los parásitos de L. dominicanus y de L. pipixcan, y significativamente diferentes que en $P$. occipitalis $(\mathrm{P}<0,0001)$. Finalmente, se observaron diferencias no significativas entre especies hospedadoras en los puntajes de las observaciones del segundo componente principal efectuados para cada sexo (machos: $\mathrm{F}_{3,24}=2,19$; $\mathrm{P}=0,11$; hembras: $\mathrm{F}_{2,30}=1,05 ; \mathrm{P}=0,36$ ).

No se encontraron diferencias significativas entre machos y hembras en los puntajes de las observaciones en los dos primeros ejes derivados del ACP, para ninguna de las especies hospedadoras (Tabla 3 ).

La fecundidad de las hembras de $P$. bullocki fluctuó en L. dominicanus entre 200.800 y 1.024 .800 huevos por hembra (mediana $=406.800)$, en L. pipixcan fluctuó entre 85.800 y 698.400 (mediana $=243.900)$, y en $P$. occipitalis entre 2.800 y 112.800 (mediana $=36.300)$. No se encontraron hembras de $P$. bullocki con huevos en $N$. phaeopus. Las mencionadas variaciones de la fecundidad de $P$. bullocki entre especies hospedadoras resultaron ser significativas $\left(\mathrm{F}_{2,27}=80,73 ; \mathrm{P}<0,001\right)$.

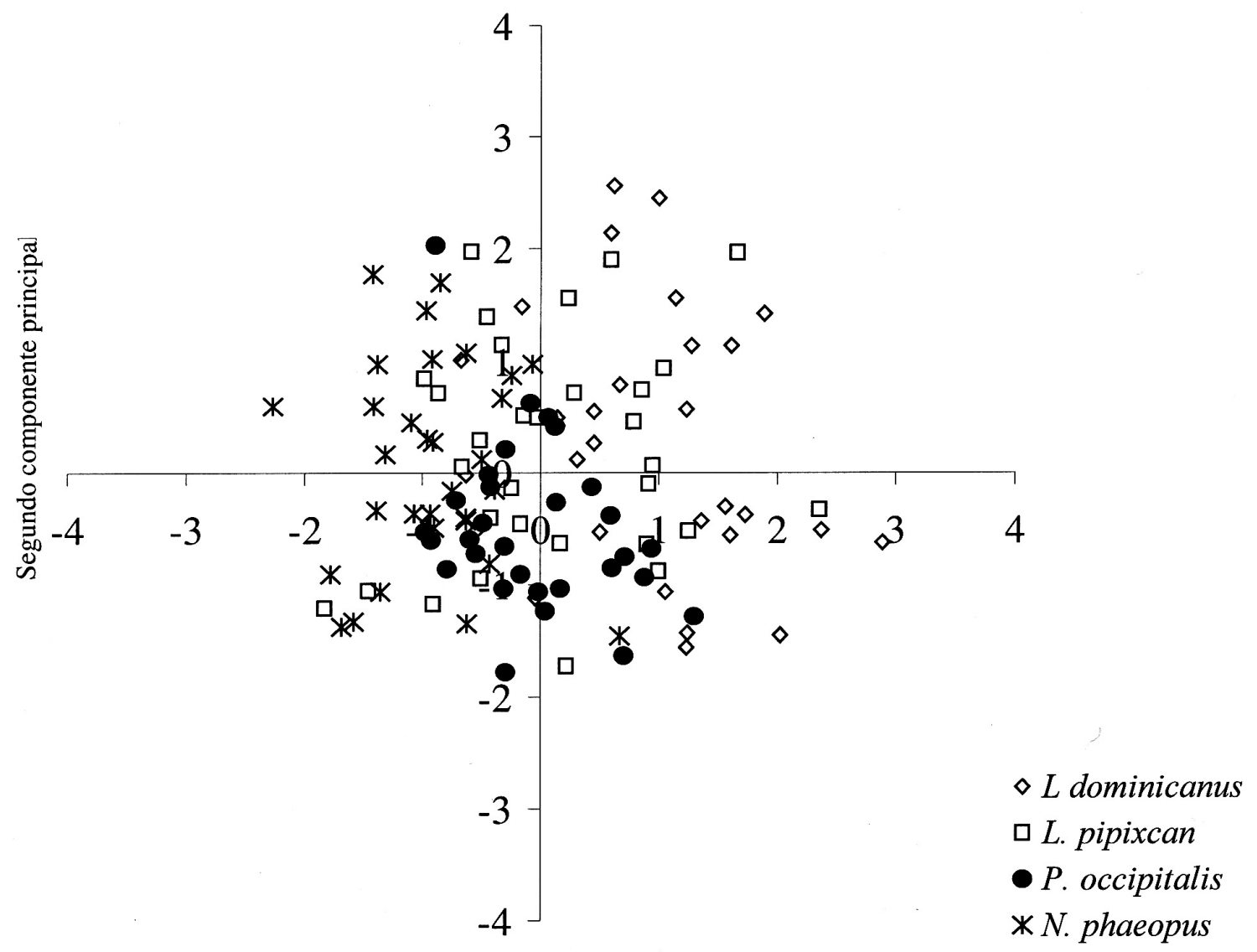

Primer componente principal

Fig. 1: Puntajes de la ordenación en los dos primeros ejes del análisis de componentes principales (ACP), realizado con nueve variables morfométricas compartidas entre machos y hembras de $P$. bullocki recolectados de $L$. dominicanus, L. pipixcan, P. occipitalis y $N$. phaeopus, en Caleta Lenga, Chile.

Scores of a PCA ordination on first 2 axes, carried out on nine morphometric variables shared by male and female $P$. bullocki collected from L. dominicanus, L. pipixcan, P. occipitalis and N. phaeopus, at Caleta Lenga, Chile. 


\section{TABLA 2}

Correlación de 16 variables morfométricas de $P$. bullocki recolectados de Larus dominicanus, $L$. pipixcan, $N$. phaeopus y $P$. occipitalis, con los puntajes de los dos primeros ejes del análisis de componentes principales llevados a cabo sobre la matriz de correlación de las variables

Correlation of 16 morphometric variables of P. bullocki collected from L.dominicanus, L. pipixcan, N.phaeopus and P. occipitalis, with the scores on the first two PCA axes carried out on their correlation matrix

\begin{tabular}{|c|c|c|c|c|c|c|}
\hline \multirow[t]{3}{*}{ Variable morfométrica } & \multicolumn{2}{|c|}{ Todos } & \multicolumn{2}{|c|}{ Machos } & \multicolumn{2}{|c|}{ Hembras } \\
\hline & \multicolumn{6}{|c|}{ Puntaje de la variable en el ACP } \\
\hline & $1^{\circ} \mathrm{CP}$ & $2^{\circ} \mathrm{CP}$ & $1^{\circ} \mathrm{CP}$ & $2^{\circ} \mathrm{CP}$ & $1^{\circ} \mathrm{CP}$ & $2^{\circ} \mathrm{CP}$ \\
\hline Longitud del cuerpo & 0,911 & 0,274 & 0,957 & 0,099 & 0,921 & 0,047 \\
\hline Largo probóscide & 0,826 & 0,135 & 0,856 & $-0,048$ & 0,798 & 0,125 \\
\hline Ancho probóscide & 0,853 & 0,036 & 0,848 & $-0,137$ & 0,781 & 0,130 \\
\hline Receptáculo de la probóscide & 0,584 & $-0,211$ & 0,580 & $-0,481$ & 0,503 & $-0,309$ \\
\hline Largo del cuello & 0,079 & 0,965 & 0,301 & 0,730 & 0,231 & 0,521 \\
\hline Ancho del cuello & 0,618 & 0,052 & 0,589 & 0,018 & 0,227 & 0,626 \\
\hline Largo del tronco & 0,928 & 0,165 & 0,955 & $-0,023$ & 0,917 & 0,068 \\
\hline Ancho del tronco & 0,830 & 0,100 & 0,857 & $-0,256$ & 0,773 & $-0,168$ \\
\hline Largo de los lemniscos & 0,763 & $-0,045$ & 0,805 & $-0,194$ & 0,644 & $-0,097$ \\
\hline Largo testículo anterior & & & 0,741 & 0,178 & & \\
\hline Largo testículo posterior & & & 0,809 & 0,143 & & \\
\hline Ancho testículo anterior & & & 0,601 & 0,018 & & \\
\hline Ancho testículo posterior & & & 0,814 & 0,077 & & \\
\hline Ancho de las glándulas de cemento & & & 0,121 & 0,776 & & \\
\hline Largo del huevo & & & & & 0,216 & $-0,484$ \\
\hline Ancho del huevo & & & & & 0,392 & $-0,610$ \\
\hline$\%$ varianza & $58 \%$ & $12 \%$ & $54 \%$ & $11 \%$ & $50 \%$ & $16 \%$ \\
\hline Número de observaciones & & & & & & 3 \\
\hline
\end{tabular}

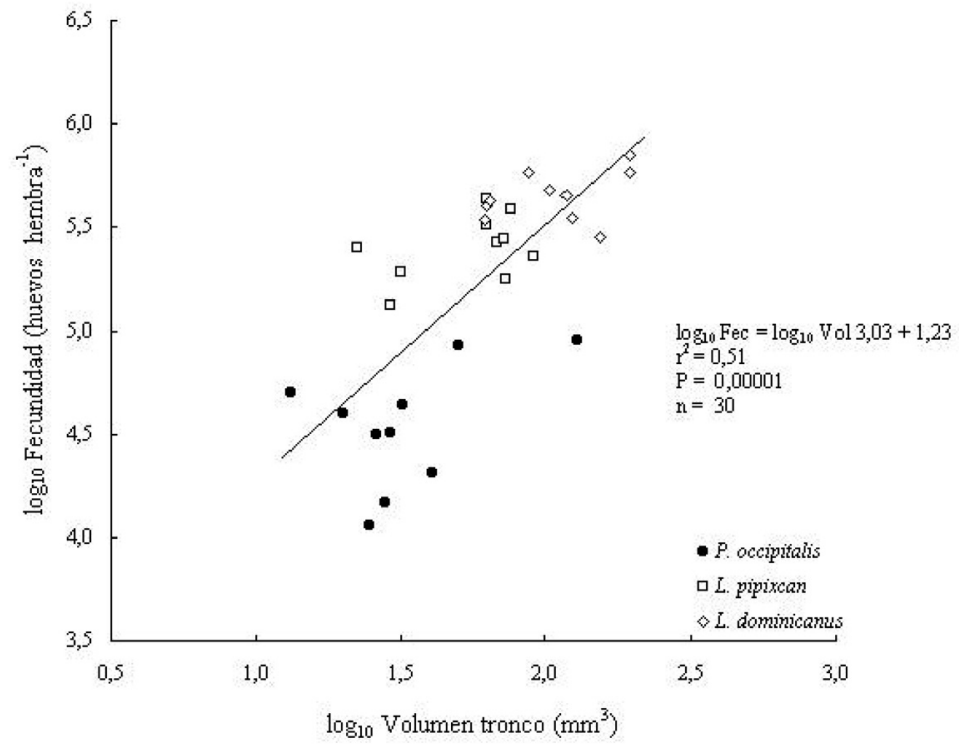

Fig. 2: Regresión lineal entre el volumen del tronco y el número huevos de las hembras del acantocéfalo $P$. bullocki en tres especies de aves $L$.dominicanus, $L$. pipixcan y $P$. occipitalis.

Linear regression between the volume of the trunk and the number of eggs per female $P$. bullocki in three host species $L$. dominicanus, L. pipixcan and P. occipitalis. 
TABLA 3

Promedio y desviación estándar de los puntajes de las observaciones morfométricas de machos y hembras del acantocéfalo $P$. bullocki, para cada especie de ave, en los dos primeros ejes derivados del ACP realizado sobre la matriz de correlación de nueve variables. Se indican los valores de t de

la prueba t de Student para la comparación entre sexos del parásito, para cada especie de ave

Arithmetic mean and standard deviation of the scores of the observations on the morphometry of male and female acanthocephalans $P$. bullocki for each bird host species, on the first two PCA axes carried out on the correlation matrix of nine variables. $t$-values from comparisons between sexes of the parasite are shown for each bird host species

\begin{tabular}{|c|c|c|c|c|c|c|c|c|c|c|}
\hline \multirow[b]{3}{*}{ Hospedador } & \multicolumn{5}{|c|}{$1^{\circ} \mathrm{CP}$} & \multicolumn{5}{|c|}{$2^{\circ} \mathrm{CP}$} \\
\hline & \multicolumn{2}{|c|}{ q } & \multicolumn{2}{|c|}{$0^{\prime \prime}$} & \multirow{2}{*}{$\begin{array}{c}\text { valor } \\
\text { de t }\end{array}$} & \multicolumn{2}{|c|}{ q } & \multicolumn{2}{|c|}{$\sigma^{\prime \prime}$} & \multirow{2}{*}{$\begin{array}{l}\text { valo } \\
\text { de } t\end{array}$} \\
\hline & Media & (DE) & Media & (DE) & & Media & (DE) & Media & (DE) & \\
\hline L. dominicanus & 0,72 & $(-0,84)$ & 0,96 & $(-0,82)$ & 0,47 & 0,51 & $(-1,41)$ & 0,16 & $(-0,51)$ & 0,47 \\
\hline L.pipixcan & 0,31 & $(-0,92)$ & 0,09 & $(-0,99)$ & 0,54 & 0,01 & $(-1,01)$ & $-0,37$ & $(-0,62)$ & 0,21 \\
\hline P. occipitalis & 0,09 & $(-0,59)$ & $-0,12$ & $(-0,56)$ & 0,33 & 0,05 & $(-0,84)$ & $-0,47$ & $(0,82)$ & 0,11 \\
\hline N.phaeopus & $-0,95$ & $(-0,79)$ & $-1,03$ & $(-0,37)$ & 0,74 & 0,17 & $(-0,82)$ & $-0,48$ & $(-1,11)$ & 0,06 \\
\hline
\end{tabular}

El tamaño de los parásitos dio cuenta del $51 \%$ de la varianza de la fecundidad (análisis de regresión simple: $\mathrm{F}_{1,28}=29,9 ; \mathrm{P}<0,00001 \mathrm{R}^{2}=$ 0,51; Fig. 2). Los residuos de dicha regresión mostraron diferencias significativas de la fecundidad entre especies hospedadoras $\left(\mathrm{F}_{1,27}=\right.$ $13,70 ; \mathrm{P}<0,0001)$. La fecundidad en $L$. dominicanus es similar a la encontrada en $L$. pipixcan y en esta especie es significativamente mayor que la encontrada en los ejemplares del acantocéfalo recolectados de P. occipitalis.

\section{DISCUSIÓN}

Aunque las asociaciones de los hospedadores y los parásitos tienden a reflejar sus relaciones evolutivas conjuntas (Hochberg et al. 1992), también, son varios los factores que afectan los procesos microevolutivos en las especies parásitas. Entre ellos están la identidad específica de la especie de hospedador intermediario y definitivo, el origen geográfico y movilidad de las poblaciones de hospedadores, la complejidad de los ciclos de vida y la densidad alcanzada en las infrapoblaciones (Hochberg et al. 1992, George- Nascimento \& Llanos 1995, Paterson $\&$ Banks 2001, Criscione et al. 2005).

Este trabajo documenta que las variaciones en morfometría (Fig. 1 y Tabla 2) y en fecundidad (Fig. 2) de P. bullocki en varias especies de aves simpátricas resultaron en que tres de las cuatro especies de aves costeras eran hospedadores definitivos y alternativos para completar el ciclo de $P$. bullocki, excepto $N$. phaeopus (Tabla 1). De ellas, las especies del género Larus serían las más adecuadas para el desarrollo de P. bullocki. Sin embargo, para establecer qué hospedadores son de mejor calidad para este parásito, es necesario considerar su abundancia, y la magnitud que alcanzan las poblaciones del parásito en cada una de ellas. Al respecto, la abundancia de los hospedadores en el sitio de estudio, aunque cambia estacionalmente producto de las migraciones, tiene el siguiente orden decreciente: L. pipixcan $>P$. occipitalis $>L$. dominicanus $>N$. phaeopus. Además, la secuencia decreciente de la prevalencia y la abundancia de $P$. bullocki es L. pipixcan $>P$. occipitalis $>N$. phaeopus $>$ L. dominicanus (L. Balboa, datos no publicados). Estos antecedentes y los resultados acerca de la fecundidad del parásito (Fig. 2) permiten sostener que, como consecuencia de la mayor abundancia del parásito y del hospedador, $P$. bullocki presentaría una mayor producción neta de huevos en L. pipixcan y P. occipitalis que en L. dominicanus.

Otras observaciones señalan que la abundancia de $P$. bullocki en el sitio de estudio aumenta cuando L. pipixcan anualmente arriba desde el hemisferio Norte (a fines de 
primavera). Por esto, su abundancia en invierno-primavera sería sostenida más por $P$. occipitalis que por L. dominicanus. Finalmente, la mayor abundancia del parásito en L. pipixcan puede deberse a una ingesta diferencial de las presas que son hospedadores intermediarios del acantocéfalo.

El escaso dimorfismo sexual encontrado en $P$. bullocki (Tabla 3) es destacable pues entre los acantocéfalos hay una amplia gama de referencias respecto a dimorfismo sexual, competencia sexual, selección sexual y uso diferencial del espacio por hembras y machos (Parker \& Partridge 1998, Sasal et al 2000, Sinisalo et al. 2004). Esto contrasta además con la mayoría de las especies parásitas dioicas que presentan dimorfismo sexual en las que frecuentemente se correlaciona, en el tamaño corporal con la fecundidad (Crompton 1985). Otras fuentes de variabilidad en el tamaño de los individuos son: el tamaño de la infrapoblación, el sitio y la edad de la infección (Sinisalo et al. 2004).

En conclusión, las expresiones del fenotipo y fecundidad de estos acantocéfalos cambian en las diferentes especies hospedadoras aun cuando provengan de una misma fuente de transmisión. Además los autores sugieren que si bien en los organismos de vida parásita la identidad de la especie de hospedador determina gran parte de las condiciones ambientales en las que ellos viven, las relaciones filogenéticas de los hospedadores, la evolución conjunta parásito-hospedador, la dieta y la tasa diferencial de ingesta de presas entre especies hospedadoras son explicaciones posibles de las variaciones en los componentes de la adecuación biológica de $P$. bullocki que son inducidas por la especie hospedadora.

\section{AGRADECIMIENTOS}

Los autores agradecen a José Pulgar y Patricio Camus por sus excelentes comentarios, a Karen González por las discusiones a lo largo del manuscrito, a dos revisores anónimos por sus sugerencias, y al laboratorio de microscopía electrónica de Universidad de Concepción. Este trabajo fue financiado por FONDECYT 2010116 a L.B., aporte del Centro de Estudios Avanzados en Ecología y Biodiversidad (CASEB), y programas 2 y 4 de la Dirección de
Investigación de la Pontificia Universidad Católica de Chile (DIPUC).

\section{LITERATURA CITADA}

AMIN O (1985) Classification. En: Crompton DWT \& BB Nickol (eds) Biology of the Acanthocephalan: 1-27. Cambridge University Press, Cambridge, United Kingdom.

ARAYA B, M BERNAL, $R$ SCHLATTER \& M SALLABERRY (1995) Lista patrón de las aves chilenas. Editorial Universitaria, Santiago, Chile. 35 pp.

ARAYA B \& G MILLIE (1992) Guía de campo de las aves de Chile. Editorial Universitaria, Santiago, Chile. $305 \mathrm{pp}$.

BAHAMONDES I \& J CASTILLA (1986) Predation of marine invertebrates by kelp gull Larus dominicanus in undisturbed intertidal rocky shores of central Chile. Revista Chilena de Historia Natural 59: 65-72.

BROCKERHOFF A \& L SMALES (2002) Profilicollis novaezelandensis n.sp. (Polymorphidae) and two other acanthocephalan parasites from shorebirds (Haematopodidae and Scolopacidae) in New Zealand, with records of two species in intertidal crabs (Decapoda: Grapsidae and Ocypodidae) Systematic Parasitology 52: 55-65.

CRISCIONE C,R POULIN \& M BLOUIN (2005) Molecular ecology of parasites: elucidating ecological microevolutionary processes. Molecular Ecology 14: 2247-2257.

CROMPTON D (1970) An ecological approach to acanthocephalan physiology. Cambridge University Press, Cambridge, United Kingdom. 125 pp.

CROMPTON D \& B NICKOL (1985) Biology of the Acanthocephala. Cambridge University Press, Cambridge, United Kingdom. 518 pp.

DUDINAK C \& V SNABEL (2001) Comparative analysis of Slovak and Czech populations of Pomphorhynchus laevis (Acanthocephala) using morphological and isoenzyme analyses. Acta Zoologica Universitatis Comenianae 44: 41-50.

GEORGE-NASCIMENTO M, M LIMA \& E ORTIZ (1992) A case of parasite-mediated competition? Phenotypic differentiation among hookworms Uncinaria sp. (Nematoda: Ancylostomatidae) in sympatric and allopatric populations of South American sea lions Otaria byronia, and fur seals Arctocephalus australis (Carnivora: Otariidae). Marine Biology 112: 527-533.

GEORGE-NASCIMENTO M \& S MARÍN (1992) Efecto de dos especies hospedadoras, el lobo fino austral Arctocephalus australis (Zimmerman) y el lobo marino común Otaria byronia (Blainville) (Carnivora; Otariidae), sobre la morfología y la fecundidad de Corynosoma sp. (Acanthocephala; Polymorphidae). Revista Chilena de Historia Natural 65: 183-193.

GEORGE-NASCIMENTO M \& A LLANOS (1995) Micro-evolutionary implications of allozymic and morphometric variations in sealworms Pseudoterranova sp. (Ascaridoidea: Anisakidae) among sympatric hosts from the southeastern Pacific Ocean. International Journal for Parasitology 25: 1163-1171

GEORGE-NASCIMENTO M \& M MUÑOZ (1997) Specificity and host suitability of Cucullanus 
genypteri (Nematoda: Cucullanidae) in three Genypterus (Ophiididae) fish host species from the southeastern Pacific Ocean. Parasite 4: 233-238.

GEORGE-NASCIMENTO M, V LOBOS, C TORRIJOS \& R KHAN (2004) Species composition of assemblages of Ceratomyxa (Myxozoa), parasites of lings Genypterus (Ophidiidae) in the southeastern Pacific Ocean: an ecomorphometric approach. Journal of Parasitology 90: 1352-1355.

GIL DE PERTIERRA A, L SPATZ \& I DOMA (1996) Systematics and metapopulation dynamics of Pomphorhynchus sphaericus n. sp. (Acanthocephala: Pomphorhynchidae) from freshwater siluriform fishes in the subtropical region of Argentina. Research and Reviews in Parasitology 56: 33-39.

HENNESSY H \& F MACHADO (1977) Acanthocephalan parasites of the sea otter, Enhydra lutris, off coastal California. California Fishing Game 63: 268-272.

HOCHBERG M, Y MICHALAKIS \& D MEEUS (1992) Parasitism as a constraint on the rate of life-history evolution. Journal of Evolutionary Biology 5: 491504.

KOELLA J, P AGNEW \& Y MICHALAKIS (1998) Coevolutionary interactions between host life histories and parasite life cycles. Parasitology 116: S47-S55.

MATEO E, R CÓRDOVA \& E GUZMÁN (1982) Polymorphus (Profilicollis) bullocki, nueva especie de acantocéfalo hallado en la gaviota Larus belcheri en el Perú. Boletín de Lima (Perú) 24: 73-78.

MATEO E, R CÓRDOVA \& E GUZMÁN (1983) Polymorphus (Profilicollis) bullocki acantocéfalo de la gaviota Larus belcheri: contribución a su ciclo biológico. Boletín de Lima (Perú) 26: 67-72.

McCLELLAND G (1980). Phocanema decipiens: Growth, reproduction and survival en seals. Experimental Parasitology 49: 175-187.

NICKOL B, D CROMPTON \& D SEARLE (1999) Reintroduction of Profilicollis Meyer, 1931, as a genus in Acanthocephala: significance of the intermediate host. Journal of Parasitology 85: 716718.

OLIVA M, J LUQUE \& A CEVALlos (1992) Parásitos de Emerita analoga (Stimpson) (Crustacea): implicancias ecológicas. Boletín de Lima (Perú) 79: 77-80.

PARKER G \& L PARTRIDGE (1998) Sexual conflict speciation. Philosophical Transactions of the Royal Society (United Kingdom) 28: 261-74.
PATERSON A \& J BANKS (2001) Analytical approaches to measuring cospeciation of hosts and parasites: through a glass, darkly. International Journal for Parasitology 31: 1012-1022.

POULIN R (1998) Evolutionary ecology of parasites: from individuals to communities. Chapman and Hall, London, United Kingdom. 209 pp.

PULGAR J, M ALDANA, E VERGARA \& M GEORGENASCIMENTO (1995) La conducta de la jaiba estuarina Hemigrapsus crenulatus (Milne-Edwards 1837) en relación al parasitismo por el acantocéfalo Profilicollis antarcticus (Zdzitowiecki 1985) en el sur de Chile. Revista Chilena de Historia Natural 68: 439-450.

SASAL P, E JOBET, E FALIEX \& S MORAND (2000) Sexual competition in an acanthocephalan parasite of fish. Parasitology 120: 65-69.

SINISALO T, R POULIN, H HOGMANDER, T JUUTI \& E VALTONEN (2004) The impact of sexual selection on Corynosoma magdaleni (Acanthocephala) infrapopulation in Saimaa ringed seals (Phoca hispida saimensis). Parasitology 128: 179-85.

SOKAL R \& F ROHLF (1995) Biometry: the principles and practice of statistics in biological research. Third edition. W.H. Freeman and Company, New York, New York, USA. 887 pp.

TANTALEÁN M, J CÁRDENAS \& R GUERE (2002) Profilicollis altmani (Perry, 1942) Van Cleave, 1947 (Acanthocephala) en el Perú. Con notas sobre la infección experimental de mamíferos terrestres. Revista Peruana de Biología 9: 49-51.

TANTALEÁN M \& J CÁRDENAS (2004) Consideración sobre Profilicollis altmani (Perry, 1942) Van Cleave, 1947 en el Perú. Revista Peruana de Biología 11: 109-111.

TANTALEÁN M, L SÁNCHEZ, L GÓMEZ \& A HUIZA (2005) Acantocéfalos del Perú. Revista Peruana de Biología 12: 83-92.

TORRES P, E RUIZ, W GESCHE \& A MONTEFUSCO (1991) Gastrointestinal helminths of fish-eating birds from Chiloé Island, Chile. Journal of Wildlife Diseases 27: 178-179.

ZDZITOWIECKI K (1985) Acanthocephalans of birds from South Shetlands (Antarctic). Acta Parasitologica Polonica 30: 11-24.

ZDZITOWIECKI K (1991) Antarctic Acanthocephala Volume 3. Synopses of the Antarctic benthos, Koenigstein, Koeltz Scientific Books, Koeningtein, Germany. $116 \mathrm{pp}$. 